\title{
Thank You, TRACK Participants
}

The Annals of Family Medicine hosts an online discussion of articles called TRACK. Our sincere thanks to the following participants in this dialogue from October 1, 2009, through September 30, 2010. To read comments or add your voice to the discussion, visit http://www.annfammed.org.

Ann Fam Med 2010;8:562. DOI: 10.1370/afm.1203.

\begin{tabular}{|c|c|c|c|c|}
\hline Jenny Advocat & Daniel J. Dutton & Richard D. Iliff & J. Lloyd Michener & Yvonne Schoon \\
\hline Christian B. Albano & Thomas R. Egnew & Steven A. Kaplan & Donald Miller & Herbert C. \\
\hline G. Caleb Alexander & Robert Eidus & David A. Katerndahl & James W. Mold & Schulberg \\
\hline Mattijs W. Alsem & Bery Engebretsen & Athanasios Katsarkas & K. G. M. Moons & Charles Schwartz \\
\hline Keri R. Anderson & Ronald M. Epstein & Aaron B. Katz & L. Gordon Moore & Robert P. Schwartz \\
\hline Jean M. Antonucci & Rhona M. Eveleigh & Robert D. Keeley & Brian Morris & Thomas L. Schwenk \\
\hline Patrick Archambault & Lyle J. (L. J.) Fagnan & Amy J. Keenum & Jane L. Murray & David Scott \\
\hline Richard L. Backman & Tom Fahey & Omar A. Khan & Ronald E. Myers & Johanna Shapiro \\
\hline Bruce Bagley & Joshua J. Fenton & Paul Kinnersley & Aanand D. Naik & Nicola T. Shaw \\
\hline Macaran A. Baird & Rich Finegan & John (Jack) W. Kirk & Richard Neill & Catherine Shisslak \\
\hline Donald A. Balasa & Timothy W. Fior & Bruno Kissling & Linda J. Niebauer & Nandi L. Siegfried \\
\hline Lynda M. Bascelli & Kevin Fiscella & Neil Korsen & Merlin Njoya & Thomas A. Sinsky \\
\hline Michael J. Bates & Oliver R. Frank & Thomas E. Kottke & Marcel Olde Rikkert & Susan M. Smith \\
\hline David W. Bauer & Andrea Franks & Roderick Kramer & Wim Opstelten & Don A. Solberg \\
\hline Carl C. Bell & Sarah Fraser & Kimberly T. Krohn & David S. Oyer & Alfred Sommer \\
\hline Ian M. Bennett & George K. Freeman & Anton J. Kuzel & David O. Parrish & Sergio Stagnaro \\
\hline Amy J. Berman & Joshua Freeman & Michel Labrecque & Christopher Pearce & Barbara Starfield \\
\hline Heiner K. Berthold & William L. Freeman & Doug J. Leedy & Marc-André Pellerin & Erin J. Starzyk \\
\hline Evan Bichara & Dan Friesner & Larry Leeman & Ted J. Phillips & G. Gayle Stephens \\
\hline Lars Bjerrum & Kathleen E. Fuller & France Legare & Michael Pignone & Richard L. Street, Jr. \\
\hline Henry Bloom & Geert-Jan Geersing & Klaus Lemke & David S. Pratt & Joachim P. Sturmberg \\
\hline Thomas & Roeland M. Geijer & Sarah E. Lesko & Richard W. Pretorius & Evelien H. Termeer \\
\hline Bodenheimer & Antonio M. Germann & Lenard I. Lesser & Nico P. Pronk & David H. Thom \\
\hline Hillary R. Bogner & James M. Gill & Robert C. Like & Dima M. Qato & Marc Tumerman \\
\hline Jeffrey Borkan & Muriel R. Gillick & Jim Little & Lee Radosh & Ross Upshur \\
\hline Howard Brody & Amanda L. Golbeck & Carl Llor & Patrick J. Raue & Johannes C. van der \\
\hline Richard L. Brown & Ralph Gonzales & Randall L. & Brian V. Reamy & Wouden \\
\hline Martha L. Bruce & Kees J. Gorter & Longenecker & Frank M. Reed & Wouter D. van Dijk \\
\hline Udo T. Buchholz & Ioanna Gouni- & Kate Lorig & Donna I. Regenstreif & Robert S. Van Howe \\
\hline Paul Buehrens & Berthold & Amanda M. Loya & Daniel J. Reidenberg & Chris van Weel \\
\hline John R. Burton & Richard W. Grant & Sean C. Lucan & Cristina Renzi & Evelyn van \\
\hline Jochen W. L. Cals & Ariel R. Green & Hilde D. P. Luijks & Hortensia Reyes & Weel-Baumgarten \\
\hline Tina Castanares & Larry A. Green & Ann C. Macaulay & Morales & Bruno Ventelou \\
\hline Anthony P. Catinella & Lee A. Green & John Mach & Robert Rhyne & Pierre Verger \\
\hline Robert M. Centor & Megan M. & Jock A. Mackenzie & Tom M. Riddle & Daniel C. Vinson \\
\hline Edoardo Cervoni & Greenberg & Harriet MacMillan & Jose O. Rivera & Jan Walker \\
\hline Wendy Chavkin & Trisha Greenhalgh & Diane J. Madlon-Kay & Claus G. Roehrborn & Emma Wallace \\
\hline Candice P. Chen & K. Allen Greiner & Eric J. Mang & James Rohrer & Lorraine S. Wallace \\
\hline Charles H. Chodroff & Paul H. Grundy & Michael W. Maples & Roger A. Rosenblatt & Samuel W. \\
\hline Carolyn Clancy & Zhonghong Guan & David Margolius & Eric S. Rovner & Warburton \\
\hline Kitty Corbett & Sylvia Guendelman & Mark R. Marnocha & Grant M. Russell & Richard C. \\
\hline Jesse C. Crosson & Calvin Gutkin & Carmel M. Martin & Nora-Mihaela Sandu & Wasserman \\
\hline Sam W. Cullison & Larry W. Halverson & Simona Mastroeni & Barry G. Saver & Robert S. Watkins \\
\hline Francois de Brantes & Tine Hansen-Turton & Jesse Matheson & Steven W. Sawczuk & Laura Weil \\
\hline George C. Denniston & M. Jawad Hashim & John F. McCarthy & Arthur Schafer & Justine P. Wu \\
\hline Dyck Dewid & Sarah T. Hawley & John M. McGrath & Bernhard Schaller & James X. Zhang \\
\hline Mathieu Doucet & Richard M. Hoffman & Heidi Meyer & Joseph E. Scherger & John A. Zweifler \\
\hline Patrick T. Dowling & Dan C. Hood & David Meyers & Henk J. Schers & \\
\hline
\end{tabular}

